\title{
Pressure dependence of ferroelectric transition temperature in TSCC
}

\author{
Authors: W.W. Windsch \& V. Hugo Schmidt
}

This is an Accepted Manuscript of an article published in Ferroelectrics in 1980, available online: http://www.tandfonline.com/10.1080/00150198008008488

"W.W. Windsch and V.H. Schmidt, "Pressure dependence of ferroelectric transition temperature in TSCC," Ferroelectrics 29, 229-234 (1980)

http://dx.doi.org/10.1080/00150198008008488

Made available through Montana State University's $\underline{\text { ScholarWorks }}$ scholarworks. montana.edu 


\title{
PRESSURE DEPENDENCE OF FERROELECTRIC TRANSITION TEMPERATURE IN TSCC
}

\author{
W. WOLFGANG WINDSCH† \\ Sektion Physik, Karl Marx Universitat, DDR-701 Leipzig, G.D.R. \\ and \\ V. HUGO SCHMIDT: \\ Department of Physics, Montana State University, Bozeman, MT 59717 U.S.A.
}

(Received March 31,1980)

\begin{abstract}
Our measured values of the ferroelectric transition temperature $T_{c}$ in tris-sarcosine calcium chloride (TSCC) for pressure $p$ between 0 and $2.1 \mathrm{kbar}$ obey the relation $T_{c}=a+b p+c p^{2}$. where $a=(128.6 \pm 0.2) \mathrm{K}, b=(+13.2 \pm 0.4) \mathrm{K} / \mathrm{kbar}$ and $c=(-0.5 \pm 0.2) \mathrm{K} /(\mathrm{kbar})^{2}$.
\end{abstract}

\section{INTRODUCTION}

Tris-sarcosine calcium chloride (TSCC) is a uniaxial ferroelectric with transition temperature $T_{c}$ between 127 and $133 \mathrm{~K},{ }^{1-5}$ depending upon crystal quality. The room-temperature structure determined from x-ray analysis by Ashida, Bando, and Kakudo ${ }^{6}$ is orthorhombic, $D_{2 h}^{16}-\mathrm{Pnma}$, but is nearly hexagonal as viewed along [100]. Crystals generally grow in a twinned form, with several ferroelastic domains which can be observed with a polarizing microscope. Sorge and Straube $^{4}$ emphasized the importance of making dielectric measurements on untwinned samples, and reported results of such measurements. From differential calorimetry they found no evidence for a second phase transition between $T_{c}$ and the decomposition temperature near $530 \mathrm{~K}$.

The structure of the ferroelectric phase has not yet been determined, but Makita ${ }^{1}$ believes it is also orthorhombic, $C_{2 v}^{9}-P n 2_{1} a$. The polarization is along [010]. Experimental evidence to date indicates a second-order transition, but a slightly first-order transition cannot be ruled out.

The Curie--Weiss-constant $C_{+}$defined by $\varepsilon_{b}=$ $\varepsilon_{\infty}+C_{+} /\left(T-T_{0}\right)$ seems to be sample dependent,

$\uparrow$ July-August, 1978, IREX visiting scholar at Montana State University.

\# On sabbatical leave $1979-80$ at Laboratoire de Spectrometrie Physique, Universite de Grenoble, B.P. n 53, 38041 Grenoble Cedex, France. but Makita's ${ }^{1}$ value of $(59 \pm 3) \mathrm{K}$ (taking the $4 \pi$ factor in his definition into account) and Sorge and Straube's ${ }^{4}$ highest value of $58 \mathrm{~K}$ are in good agreement.

The spontaneous polarization as measured by Makita ${ }^{1}$ is near $0.27 \mu \mathrm{Ccm}^{-1}$ at $78 \mathrm{~K}$ but is still increasing fairly rapidly with decreasing temperature at that temperature. Sorge and Straube ${ }^{4}$ report similar behaviour but with a more rounded curve of $P_{s}$ vs. $T$. Both have analtzed their susceptibility and spontaneous polarization results in terms of a Landau free energy expansion

$G=\frac{1}{2} A_{0}\left(T-T_{0}\right) P^{2}+\frac{1}{4} B P^{4}+\frac{1}{6} C P^{6}$

and find quite similar $A_{0}$ and $B$ coefficients, but Makita did not include the 1/6CP $C P^{6}$ term.

Windsch et al. ${ }^{2}$ have shown that the EPR (electron paramagnetic resonance) fine-structure tensor of $\mathrm{Mn}^{2+}$ ions on $\mathrm{Ca}^{2+}$ sites with a concentration of $10^{-5}$ splits into two tensors below $T_{c}$. rotated $2 \alpha$ apart from each other with $\alpha$ being proportional in lowest order to the spontaneous polarization $P_{s}$. They determined an effective critical exponent $\beta(T)$ according to the relation

$\alpha(T)=\alpha_{g} \varepsilon^{\beta(T)}$

where $\varepsilon=\left(T_{0}-T\right) / T_{0}$ and $\alpha_{g}$ is the rotation angle at $0 \mathrm{~K}$. At $4.2 \mathrm{~K}$ they measured $\alpha_{g}=31.5^{\circ}$. They found $B(T)$ decreasing from 0.35 at $T_{\mathrm{c}}-1 \mathrm{~K}$ to 0.22 at $T_{c}-22 \mathrm{~K}$. 
The temperature dependence of this effective exponent of the order-parameter was explained by Franck et al. ${ }^{7}$ by the influence of noncritical fluctuations on the coefficients $B$ in the fourth order term of the Landau expansion (1). The coefficient $B$ should show a linear temperature dependence and change its sign at about $12 \mathrm{~K}$ below $T_{c}$. This proposed linear temperature dependence of $B$ could not be verified by means of dielectric methods, but by using the temperature independent coefficients $A_{0}, B, C$ given by Sorge and Straube ${ }^{4}$ it can be shown that $\beta(T)=0.5$ is valid only in the limit $T \rightarrow T_{\mathrm{c}}$ and that $\beta(T) \simeq 0.3$ for $T_{c}-T \geq 5 \mathrm{~K}$.

Information regarding the nature of the transition, whether order-disorder or displacive, is best obtained from dynamic measurements. Measurements of EPR linewidth and spin-lattice relaxation $^{8-10}$ have been interpreted in terms of an order-disorder transition. Recent Raman scattering studies revealed a soft mode below $T_{c}^{11,12}$ which indicates that the transition is at least partly displacive.

The purpose of this paper is to present in more detail our previously reported ${ }^{13}$ study of the effect of hydrostatic pressure up to $2.1 \mathrm{kbar}$ on the transition temperature of TSCC. The following section deals with sample preparation and apparatus. Then the experimental method is presented, followed by the experimental results. The paper concludes with an analysis of the experimental and findings.

\section{EXPERIMENTAL METHODS AND RESULTS}

The crystals were grown from a water solution of sarcosine and $\mathrm{CaCl}_{2}$ in stoichometric ratio by slow evaporation near room temperature. For the measurements, a ferroelastic monodomain specimen was selected from a twinned crystal using a polarizing microscope.

The crystal and its holder were placed in the the cavity of a beryllium-copper pressure vessel. This vessel is surrounded by a copper can, which in turn is surrounded by a brass can immersed in liquid nitrogen. The cans are attached only to a 0.25 in. stainless steel pressure tubing which is coaxial with the cans and pressure vessel. The space between the pressure vessel and brass can is evacuated. Heaters are provided for both the pressure vessel and copper can. In normal operation these heaters keep the can only a few degrees colder than the pressure vessel, so that the can acts as a thermal radiation shield and minimizes temperature gradients within the pressure vessel. The temperatures of the pressure vessel and copper can were monitored with copper constantan thermocouples using an ice bath reference.

The source of high pressure fluid was a bottle of helium gas. Its pressure was increased first by a remote head and then by a 10:1 intensifier, both of which were operated by means of oil pressurized by a hand pump. A manganin cell and associated bridge were used to monitor the pressure. Details of the pressure and temperature control system appear elsewhere. ${ }^{14}$

Our electrical measurements were made by an ac bridge method with a Model HR-8 Princeton Applied Research Phase Sensitive Detector serving both as power source and detector. It supplied $1000 \mathrm{~Hz}, 0.02 \mathrm{~V}_{\mathrm{ms}}$ ac to the bridge. One branch of the bridge consisted of the crystal in series with a $0.001 \mu f$ capacitor, while the other branch was a high $Q$ ratio transformer. Accordingly the two branches of the bridge are capacitive and inductive dividers respectively. The two dividing points were connected to the $A$ and $B$ inputs of the type $\mathrm{A}$ preamplifier of the HR-8, which was operated in the $A-B$ mode as a null detector. No phase adjustment circuit was necessary. Normally we would have run two ultraminiature coaxial cables down the $0.0625 \mathrm{in}$. inside diameter pressure tubing, with center conductors connected to each side of the crystal and shields grounded to minimize stray capacitance in parallel with the crystal.

When we began this experiment, however, one cable was broken and could not be replaced in time. We were forced to use an arrangement having a large cable capacitance in parallel with the crystal. We were still able to locate the peak of the dielectric constant accurately as a function of temperature, but could not obtain absolute values of dielectric constant or Curie-Weiss constant.

Electrical contact was obtained by application of silver paint to the entire surface on each side of the crystal, and placing the crystal in a small leaf-spring holder. We controlled the temperature by varying the liquid nitrogen level. The resulting rate of temperature change was about $1 \mathrm{~K}$ per hour.

We made measurements at seven pressures, four while warming the crystal, one while cooling, and two both warming and cooling. After reopening the vessel we saw that the silver paint contacts has separated from the crystal; this was obviously caused by the high compressibility of 
the TSCC crystal. This separation caused some anomalous readings near the end of our measurement series, but did not prevent us from finding the dielectric peaks.

The dielectric constant of the crystal is proportional to $r-r_{0}$, where $r$ is the ratio transformer setting for bridge balance and $r_{0}$ is subtracted to account for the capacitance in parallel with the crystal as well as that part of the crystal capacitance not associated with the transition mechanism. A plot of $\left(r-r_{0}\right)^{-1}$ as a function of temperature for the pressure $p=2.082 \mathrm{kbar}$ is shown in Figure 1. For each pressure, that value of $r_{0}$ was chosen which gives straight lines for the plot. (A variation of $r_{0}$ with pressure is expected because the cable in

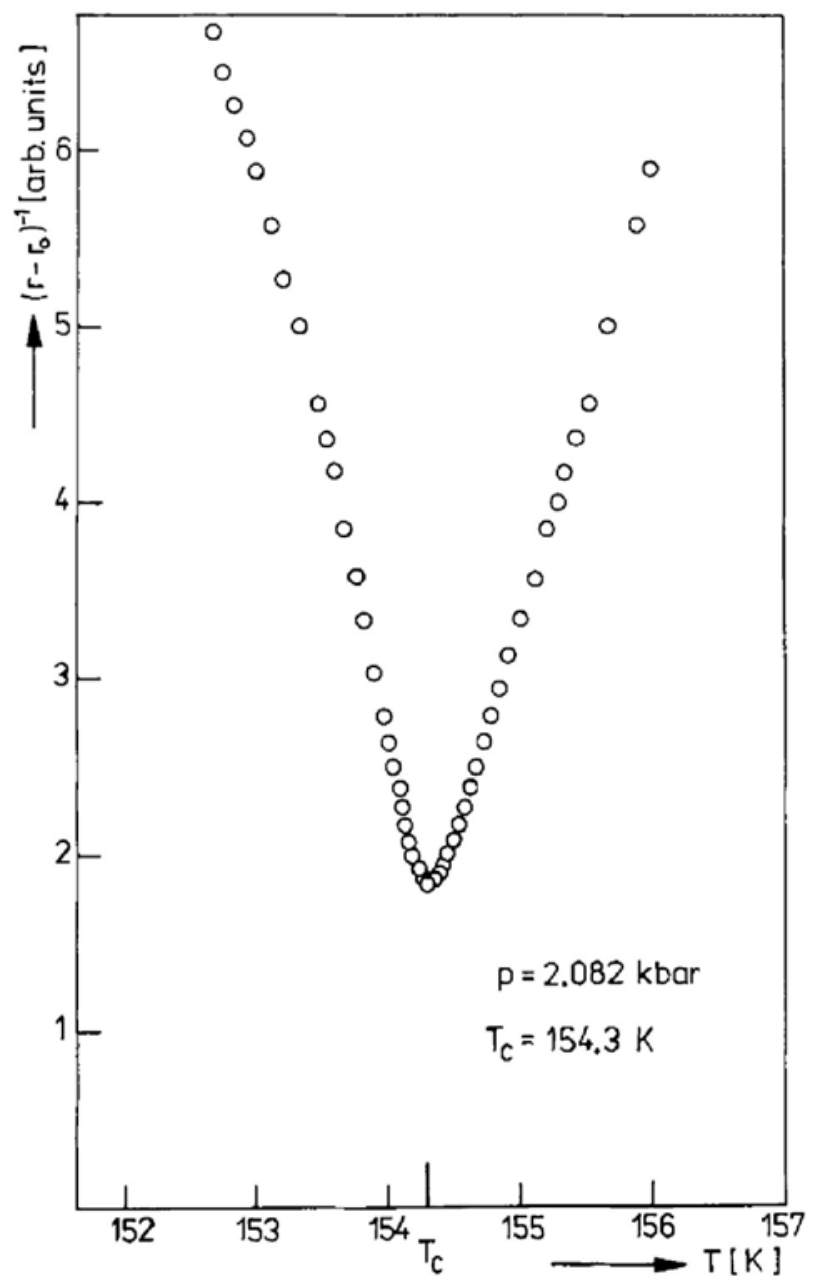

FIGURE I Inverse dielectric permittivity (in units of ratio transformer settings for bridge balance) vs. temperature for hydrostatic pressure $p=2.082 \mathrm{kbar}$.

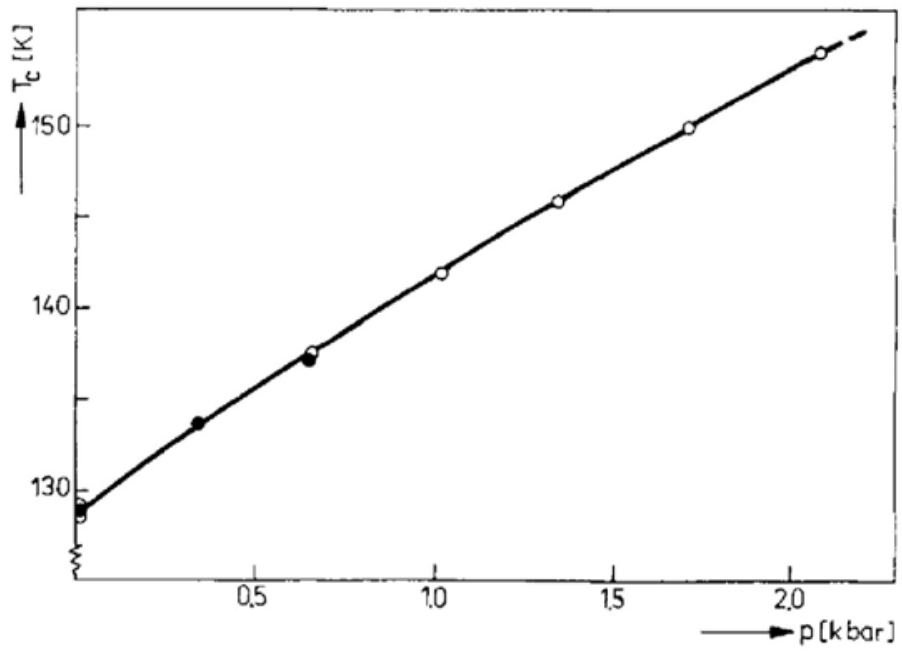

FIGURE 2 Transition temperature vs, hydrostatic pressure $\square$ Increasing temperature run. $\square$ Decreasing temperature run.

parallel with the crystal is inside the pressure tubing).

There is no definite trend of dielectric constant with pressure. The intersection of the lines for values of $\left(r-r_{0}\right)^{-1}$ seems to indicate a first-order transition, but experience with TGS $^{15}$ has shown that if the crystal has imperfections, such an erroneous indication of a first-order transition can occur.

Our results for these warming and cooling runs are shown in Figure 2. The pressure dependence of $T_{c}$ can be represented by the formula

$T_{c}(p)=T_{c}(0)+p \frac{d T_{c}}{d p}+p^{2} \frac{d^{2} T_{c}}{d p^{2}}+\cdots$

The values for the three coefficients above are

$T_{c}(0)=(128.8 \pm 0.2) \mathrm{K}$

$\frac{d T_{c}}{d p}=(+13.2 \pm 0.4) \mathrm{K}(\mathrm{kbar})^{-1}$

$\frac{d^{2} T}{d p^{2}}=(-0.5 \pm 0.2) \mathrm{K}(\mathrm{kbar})^{-2}$

The Curie-Weiss law for the dielectric constants holds over the whole pressure range investigated. The ratio of the Curie constants $C_{+} / C_{-}$deviates strongly from the value predicted by Landau theory. At zero pressure it is 1.14 and increases with pressure at a ratio $d\left(C_{+} / C_{-}\right) / d p=0.17 \mathrm{kbar}^{-1}$. The Curie constant $C_{+}$for the paraelectric phase is pressure independent whereas $C_{-}$for the ferroelectric phase decreases with pressure. 


\section{DISCUSSION}

The most striking feature of the pressure dependence of $T_{c}$ in TSCC is its rapid increase with pressure. The material which resembles TSCC most closely with regard to having low $T_{c}$ and large positive $d T_{c} / d p$ is $\mathrm{RbHSO}_{4}$ with $T_{\mathrm{c}}$ at $264 \mathrm{~K}$ and $d T_{c} / d p=12.0 \mathrm{~K} / \mathrm{kbar}^{16}{ }^{16}$ Its structure may have disordered sulfate groups above $T_{c},{ }^{17}$ whose ordering may be responsible for the transition. ${ }^{18}$ Other crystals such as Rochelle salt and TGS-type compounds with rotationally disordered elements which order at $T_{\mathrm{c}}$ also tend to have positive $d T_{\mathrm{c}} / d p .^{19}$

Crystals such as $\mathrm{KH}_{2} \mathrm{PO}_{4}$ in which hydrogen intrabond ordering is responsible for the transition have negative $d T_{c} / d p,{ }^{19}$ but TSCC is not likely to be in this group because its hydrogen bonds are of the $\mathrm{N}-\mathrm{H} \ldots \mathrm{Cl}$ type $^{6}$ and are probably too asymmetric to become disordered.

The above evidence is consistent with TSCC having an order-disorder transition not triggered by hydrogen bonds. Also, EPR results ${ }^{10}$ have been interpreted in terms of an order-disorder transition. However, recent Raman results. ${ }^{11,12}$ indicate that the transition is at least partly displacive in nature. Further structural studies are needed to determine the nature of the transition. Another feature of the pressure dependence of $T_{c}$ observed both by us ${ }^{13}$ and by Hegenbarth and Schmidt ${ }^{5}$ is a significant decrease in $d T_{c} / d p$ with increasing pressure over the fairly narrow pressure range (4.2 kbar) investigated.

Similar nonlinearities were also observed in TGS. $^{20,21}$ According to Klimowski and Wanarski ${ }^{22}$ such a nonlinear relation between the shift of the transition temperature and the hydrostatic pressure may be due to the pressure dependence of the electrochemical coupling coefficient $k$. The coefficient of the second order term in the Landau free energy expansion (1) may be written

$A=\frac{1}{C_{+}}\left[T-T_{c}(0)\right]-K p$

with

$K=K_{0}+K^{\prime}\left[T-T_{c}(0)\right]+K^{\prime \prime} p^{2}$.

Usually $K^{\prime}\left[T-T_{c}(0)\right] \ll K^{\prime \prime} p$ holds $^{21}$ and we obtain

$T_{c}(p)=T_{c}(0)+C_{+}\left(K_{0} p+K^{\prime \prime} p^{2}\right)$

Our measured values result in

$k_{0}=3.1(\mathrm{kbar})^{-1}, k^{\prime \prime}=-0.21(\mathrm{kbar})^{-2}$
A comparison with the values for $\mathrm{TGS}^{21} k_{0}=$ $8.1 \cdot 10^{-3}(\mathrm{kbar})^{-1}, k^{\prime \prime}=-0.18 \cdot 10^{-3}(\mathrm{kbar})^{-2}$ points to a very strong electromechanical coupling in TSCC which may be due to its ferroelastic properties. $^{23}$

The decrease of the deviation of $C_{+} / C_{-}$from its value expected from Landau theory with increasing pressure is not yet possible to explain. Dielectric investigation on TSCC with different lattice defects ${ }^{24}$ show for good quality pure crystals $C_{+} / C_{-} \simeq 2$. $C_{+}$is only weakly dependent on defect concentration whereas $C_{-}$increases markedly with increasing number of lattice defects. Our investigations indicate that our crystal had many such defects, and that lattice defects have a smaller effect on ferroelectric properties as pressure increases.

Recently, Hegenbarth and Schmidt ${ }^{5}$ measured the dielectric susceptibility of TSCC at pressures up to $4.2 \mathrm{kbar}$ and noted a quite rapid disappearance of the dielectric anomally with increasing pressure near $4 \mathrm{kbar}$ and $180 \mathrm{~K}$. This indicated the existence of a third phase, whose properties we are currently investigating. ${ }^{25}$

\section{ACKNOWLEDGEMENTS}

The authors are grateful for experimental assistance by P. T. Schnackenberg and helpful discussions with Prof. J. F. Scott, and to B. Malige for growing the crystals. The work was supported by National Science Foundation Grant DMR7809205 and by the International Research and Exchanges Board (IREX).

\section{REFERENCES}

1. Y. Makita. J. Phys. Soc. Japan, 20, 2073 (1965).

2. W. Windsch, R. Lippe, and G. Völkel, Solid State Commun., 17. 1735 (1975)

3. A. Levstik, C. Filipič, and R. Blinc, Solid State Commun., 18. 1231 (1976).

4. G. Sorge and U. Straube, Phys. Stat. Sol., (a) 51, 117 (1979).

5. E. Hegenbarth and B. Schmidt, Ferroelectrics, 25, 359 (1980).

6. T. Ashida, S. Bando, and M. Kakudo, Acta Cryst., B28. 1560 (1972).

7. S. Franck, A. Kühnel, T. Nattermann, and S. Wendt, Phys. Siat. Sol. (b) 77,631 (1976).

8. R. Lippe. W. Windsch, G. Völkel, and W. Schulga, Solid State Commun., 19, 587 (1976).

9. G. Völkel, W. Brunner, and W. Windsch, Solid State Commum., 17, 345 (1975).

10. W. Windsch and G. Völkel, Ferroelectrics, 24, 195 (1980).

11. S. D. Prokhorova, G. A. Smolensky, I. G. Siny, E. G. Kuzminov, V. D. Mikvabya, and H. Arndt, Ferroelectrics, 25, 629 (1980). 
12. G. E. Feldkamp, K. Douglas, B. B. Lavrenčič, and J. F. Scott, Bull. Am. Phys. Soc., 25, 171 (1980)

13. W. Windsch and V. H. Schmidt, Bull. Am. Phys. Soc., 24, 507 (1979).

14. A. B. Western, A. G. Baker, C. R. Bacon, and V. H. Schmidt, Phys. Rev., B17, 4461 (1978).

15. K. Okada. J. A. Gonzalo, and J. M. Rivera, J. Phys. Chem. Solids. 28. 689 (1967).

16. K. Gesi and K. Ozawa, J. Phys. Soc. Japan. 38, 459 (1975). (1975).

17. J. P. Ashmore and H. E. Petch, Canad. J. Phys., 53, 2694 (1975).

18. D. H. Blat and V. I. Zinenko, Sotiet Phys.-Solid State, 18, 2096 (1976).
19. G. A. Samara, J. Phys. Soc. Japan, 28S, 399 (1970).

20. J.Stankowski, A. Galezewski, S. Waplak, U. Gruszczynska, and H. Gierszal, Ferroelectrics, 6, 209 (1974).

21. J. Klimowski, K. Holderna, W. Wanarski, R. Makowski. D. Ozgo, and R. Konkol, Phys. Stat. Sol., (a) 20, K61 (1973).

22. J. Klimowski and W. Wanarski, Acta Phys. Pol., A45, 203 (1974).

23. A. Sawada, Y. Makita, and Y. Takagi, J. Phys. Soc. Jap.. 42. 1918 (1977).

24. H. Schlemmbach, W. Windsch, and S. Heinig, Acta Universitatis Wratislaviensis (in press)

25. V. H. Schmidt, to appear in Solid State Commun., 30. 
